\title{
Archéopages
}

Archéopages

Archéologie et société

37 | 04/2013

Jardins

\section{Dolia, le système documentaire de l'Inrap. État des lieux et perspective}

Emmanuelle Bryas, Gilles Bellan, Anne Speller et Carine Carpentier

\section{(2) OpenEdition}

1 Journals

Édition électronique

URL : https://journals.openedition.org/archeopages/357

DOI : 10.4000/archeopages.357

ISSN : 2269-9872

Éditeur

INRAP - Institut national de recherches archéologiques préventives

Édition imprimée

Date de publication : 1 février 2014

Pagination : $72-81$

ISSN : 1622-8545

\section{Référence électronique}

Emmanuelle Bryas, Gilles Bellan, Anne Speller et Carine Carpentier, «Dolia, le système documentaire de l'Inrap. État des lieux et perspective », Archéopages [En ligne], 37 | 04/2013, mis en ligne le 01 avril 2015, consulté le 22 janvier 2022. URL : http://journals.openedition.org/archeopages/357 ; DOI :

https://doi.org/10.4000/archeopages.357 
1

a

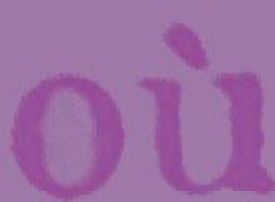

vin,

êt?

to

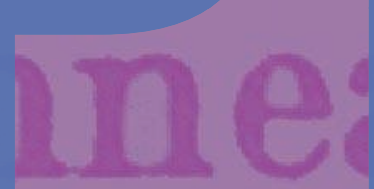

I'huile,

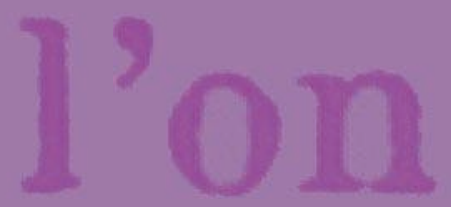

ou

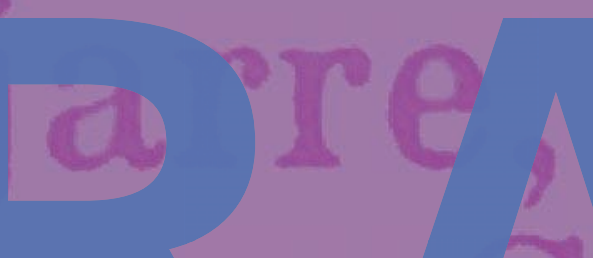

$\Leftrightarrow$

ค 2 n.

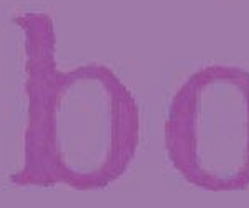
serrait
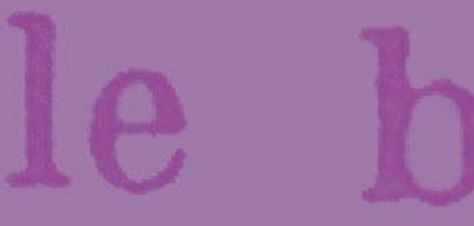

429
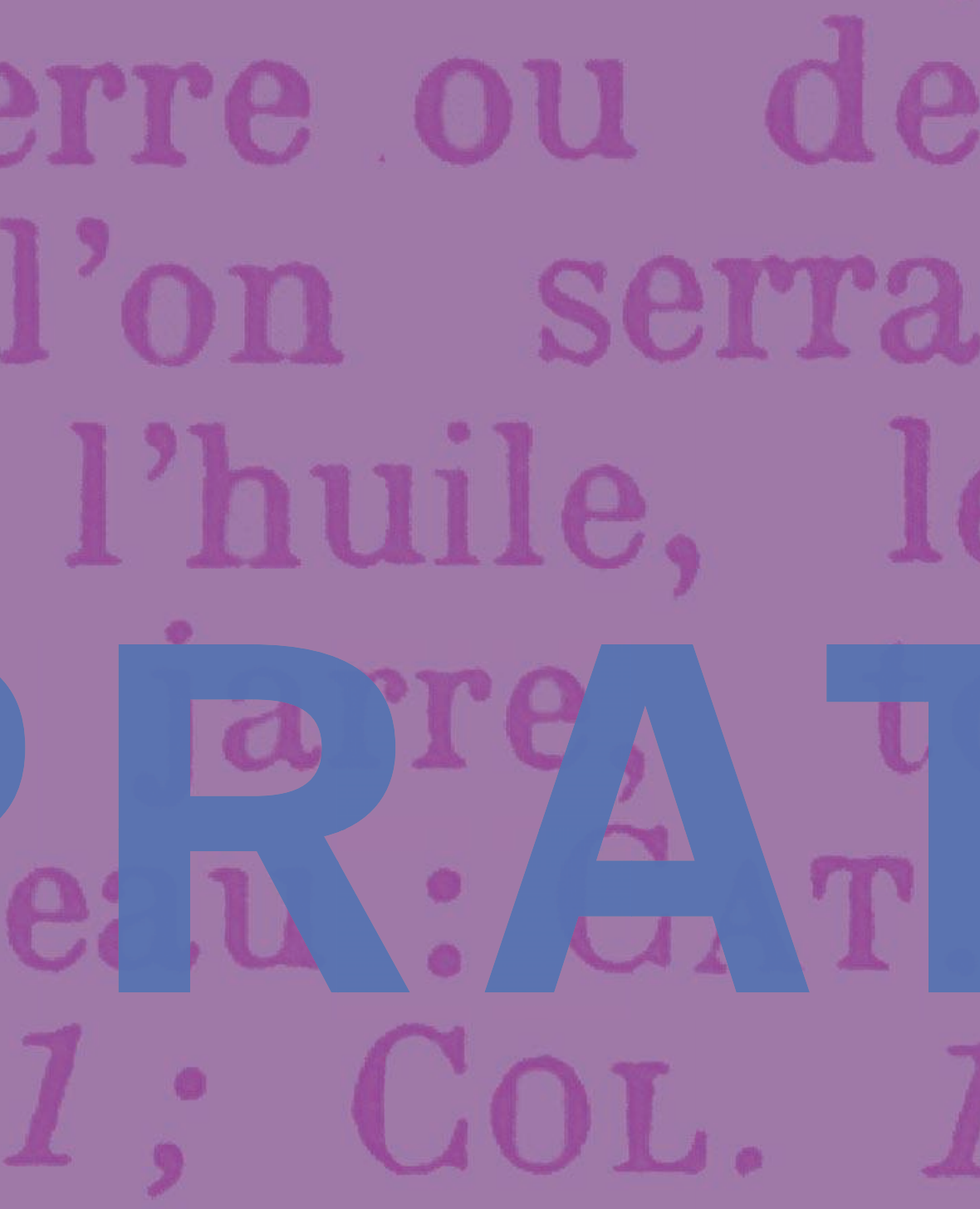

un.
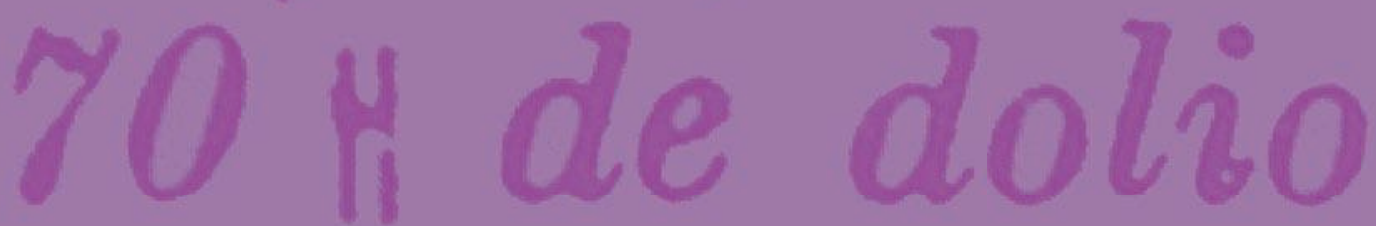

ha
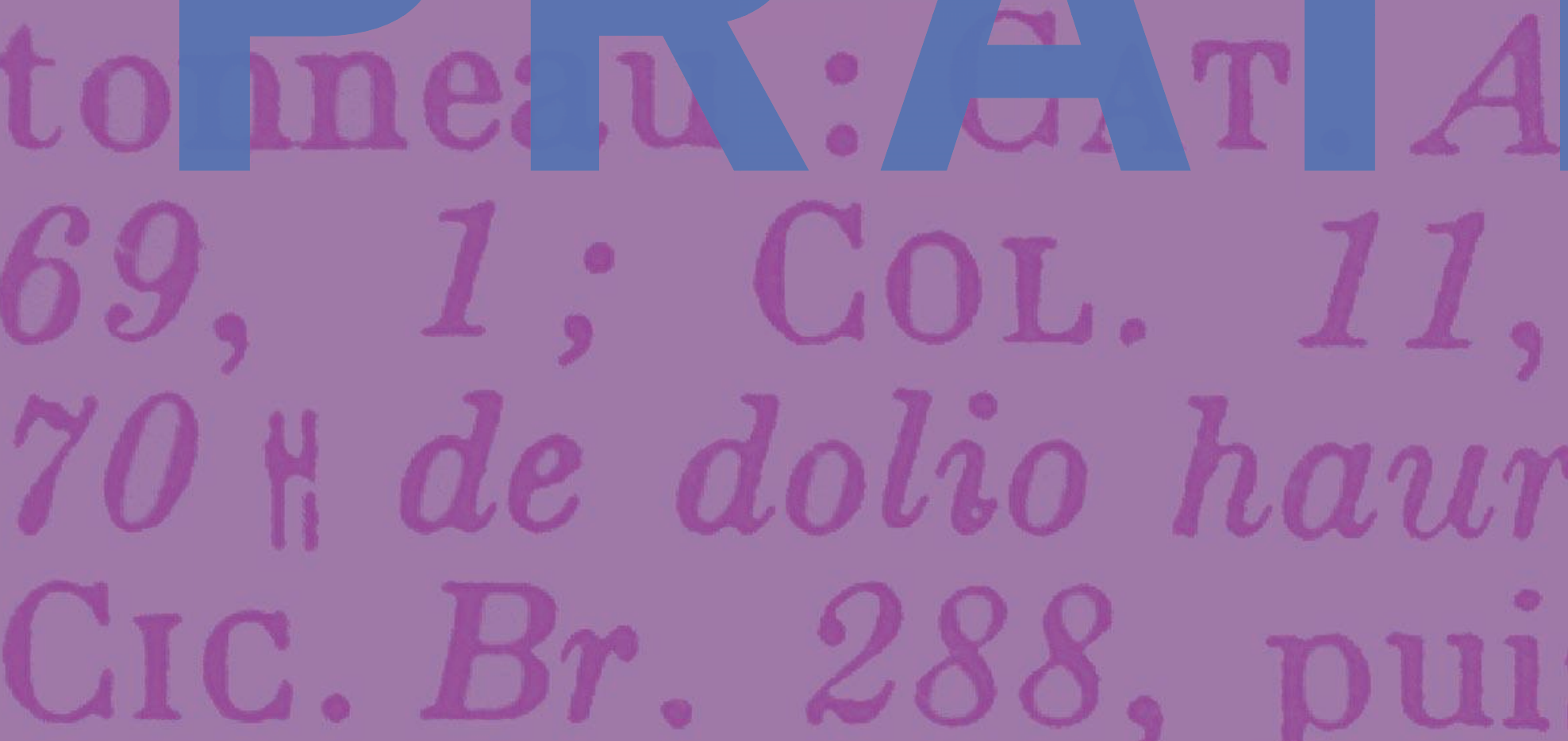

,

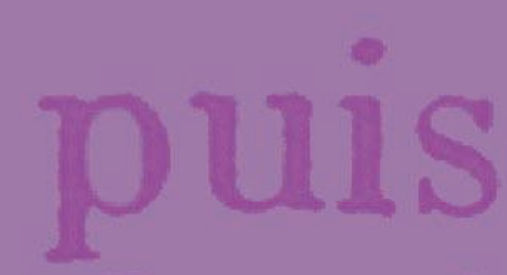

au

tonneau

Q 11

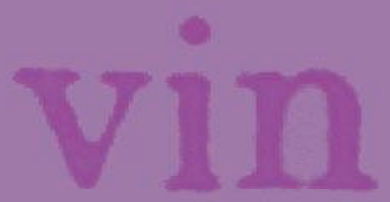

de

1

'ann

é

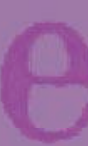




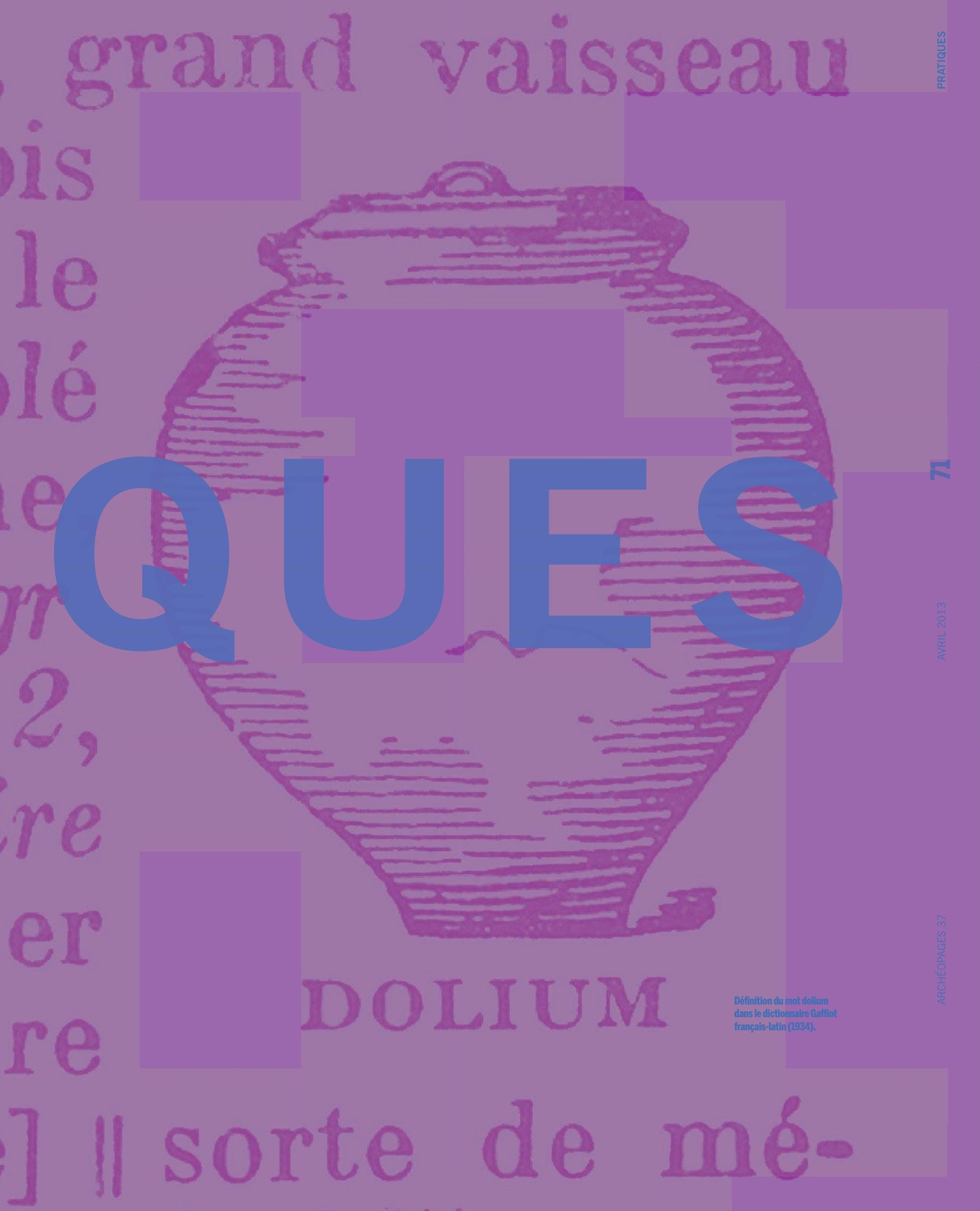




\title{
Dolia, le système documentaire
}

de l'Inrap

État des lieux et perspective

\author{
Emmanuelle Bryas Irrap \\ Gilles Bellan Inrap \\ Anne Speller Inrap \\ Carine Carpentier Inrap
}

L'enjeu que constitue le partage des données scientifiques issues des opérations archéologiques a conduit l'Inrap à mettre en œuvre en 2006 un système d'information permettant, notamment, l'exploitation des rapports d'opération et des archives

\section{Les fonds de l'Inrap}

C'est à partir de 2008 que les fonds documentaires de l'Inrap ont été systématiquement constitués, classés, répertoriés alors que simultanément s'ouvrait le catalogue documentaire scientifique de l'Inrap [ill. 1] - Dolia - et qu'un réseau de documentalistes ${ }^{1}$ entrait en fonction ${ }^{2}$ [ill. 2]. Il fallut alors rassembler des centaines de documents (littérature grise ou ouvrages) déposés dans les bases archéologiques ou acquis au gré des opérations. Au-delà, il s'agissait aussi de mettre en place une politique d'achat d'ouvrages de fond (monographies et périodiques) et d'usuels (dictionnaires, manuels, catalogues) afin d'en doter les centres archéologiques, de répondre aux besoins permanents des archéologues, notamment par un service de prêt national. Ce service documentaire, répondant à des besoins immédiats, ne venait pas pour autant doubler les bibliothèques des multiples partenaires avec lesquelles s'installaient des collaborations (services du Patrimoine, centres de recherche du CNRS, des collectivités territoriales ou de l'Université). De même, il ne s'agissait pas, en gérant une partie de la documentation produite par les opérations archéologiques, de se substituer à la mission de conservation archivistique assurée par l'État. de fouille. Sept ans plus tard, si les objectifs initiaux paraissent remplis, les technologies numériques imposent de nouveaux défis à l'ensemble des acteurs de l'archéologie préventive et plus particulièrement aux acteurs du secteur documentaire.
1. Désignés à l'Inrap sou le nom de gestionnaires de documentation. 2. Projet mené par la direction scientifique et technique de l'Inrap.
Trois objectifs essentiels étaient poursuivis : créer pour les archéologues de l'Inrap un fonds d'ouvrages de référence; permettre, en organisant à l'échelle nationale la conservation et la diffusion de la documentation produite lors des opérations de terrain, une mutualisation des résultats des recherches archéologiques menées au sein de létablissement ; et enfin contribuer à diffuser les travaux des archéologues de l'Inrap.

Partant d'un dispositif documentaire quasi inexistant, l'Inrap s'est donc doté en quelques années d'un système d'information mis à la disposition du public, d'un réseau de documentalistes et de locaux adaptés. Mais l'axe fort de cette politique a été surtout de prendre à bras le corps le corpus des rapports d'opération, dont il faut rappeler que la majorité sont produits par les agents de l'Inrap.

\section{Rapports d'opération, littérature grise}

Il était évident, dès l'origine du projet, que le rapport d'opération était - et reste - la ressource documentaire réclamant priorité de traitement. Tout d'abord en raison de l'usage fréquent que les archéologues sont amenés à en faire, lorsqu'il s'agit quotidiennement de « documenter » telle ou telle région, tel ou tel terroir en prévision de nouvelles 
1. Dolia en chiffres.

a. Répartition des inscrits à Dolia (hors Inrap). Il est demandé à tout nouveau

lecteur s'inscrivant à Dolia de renseigner l'institution à laquelle il appartient et de

la classer dans une des douze catégories reprises dans

le graphique ci-dessus.

La catégorie « aucune

institution $)$ regroupe

des lecteurs qui n'ont pas

souhaité transmettre cette

information ou n'ont pas

su classer leur institution

dans une des onze autres

catégories.

b. Répartition des notices

dans le catalogue par nature

de document. Chaque année,

l'Inrap réalise environ deux

mille diagnostics pour plus

de deux cents fouilles. Les

délais de rendu des rapports

de diagnostic sont plus courts

que ceux des rapports de

fouilles. La conjonction de

ces deux éléments explique

la part écrasante des notices

de rapport de diagnostic

dans le catalogue Dolia.

c. Répartition régionale

des rapports catalogués

dans Dolia. Ce graphique est

moins le reflet de l'activité

archéologique - si ce n'est

le ratio diagnostic/fouille -

qu'un état des fonds

documentaires par nature

de rapport d'opération.
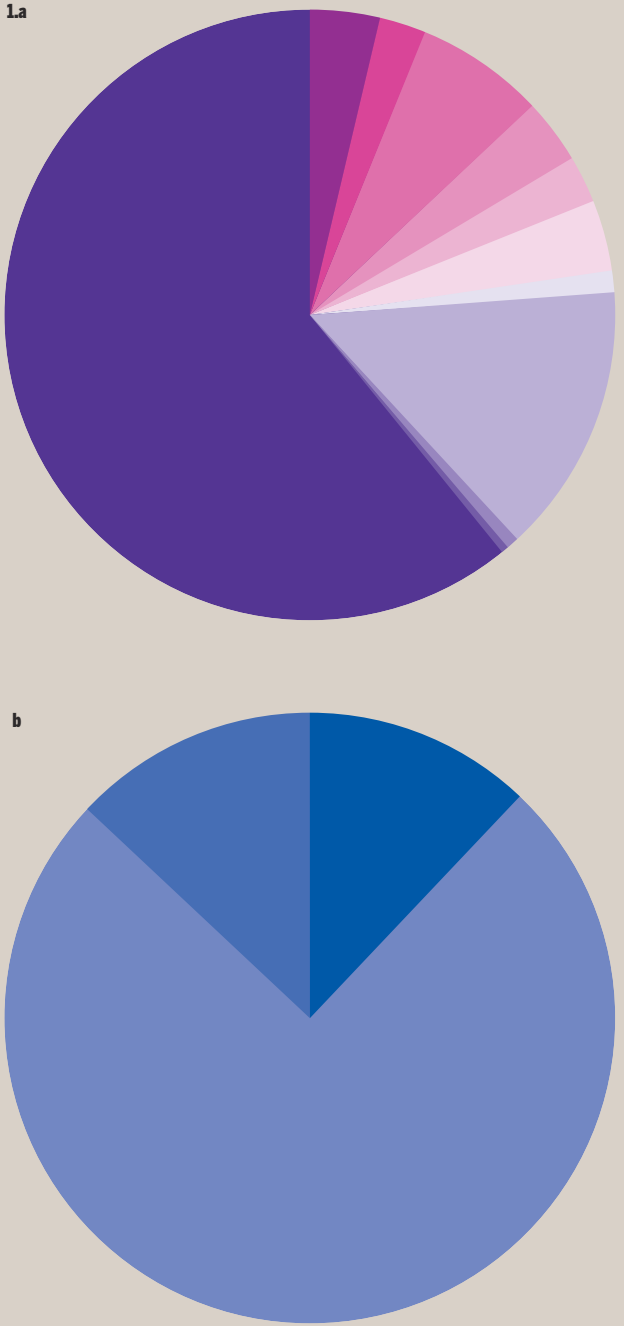

Établissement d'enseignement primaire et secondaire $=0,1 \%$ CNRS

Ministère de la Culture

Collectivité territoriale

Opérateur d'archéologie agréé

Établissement étranger

Association

Musée, parc archéologique

Établissement d'enseignement supérieur Laboratoire, société, bureau d'études

Institut, organisme public $=0,2 \%$

Aucune institution
Rapports de fouilles (12\%)

Rapports de diagnostic (75\%)

Livres édités, périodiques (13\%)

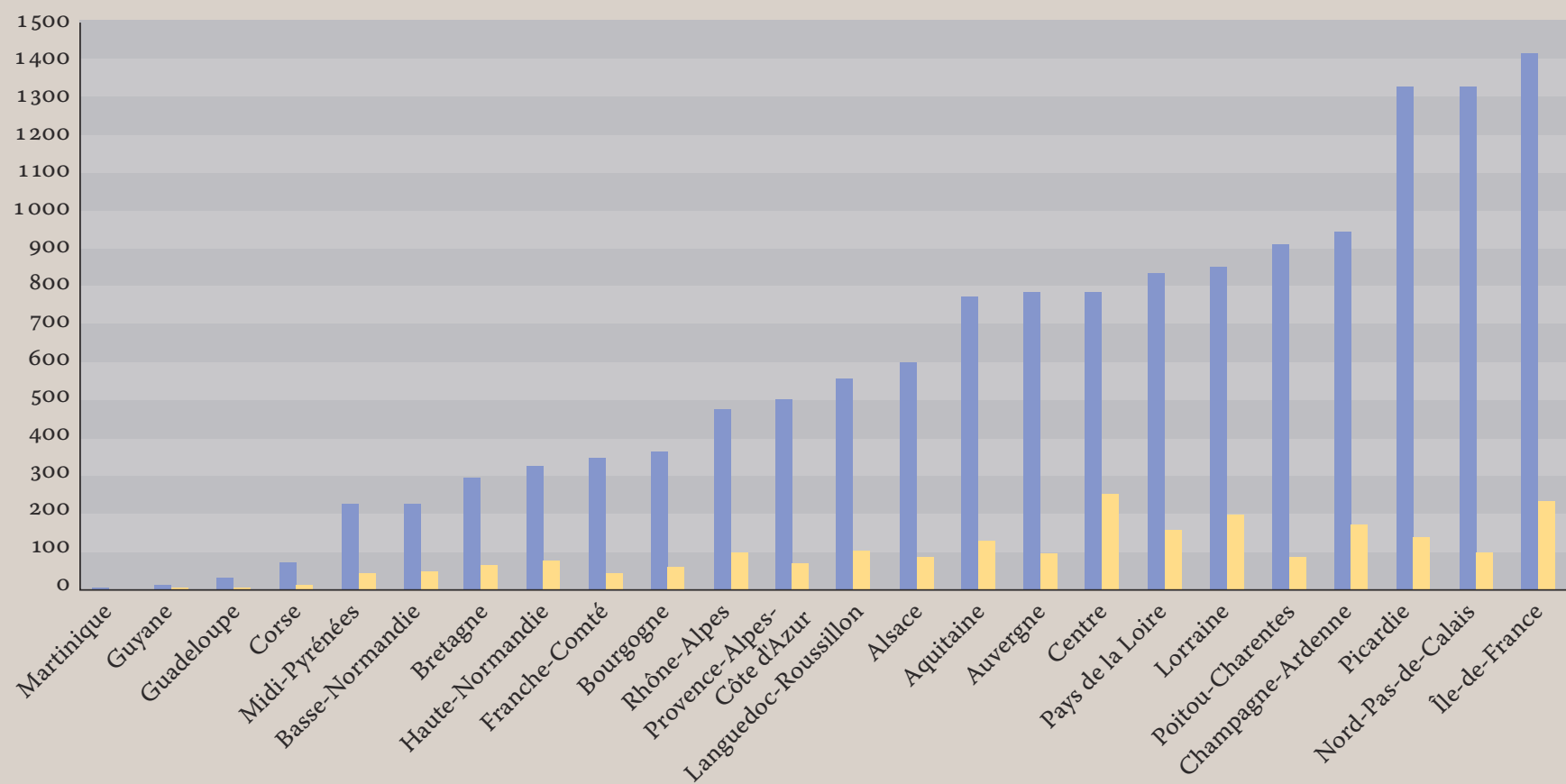


2. Carte de la répartition géographique des centres de documentation. Treize documentalistes composent le réseau documentaire de l'Inrap. Ils sont affectés dans des centres archéologiques qui couvrent l'ensemble du territoire métropolitain. Outre les collections de leur centre d'affectation, ils peuvent avoir en charge celles des centres

archéologiques de l'interrégion. Corollairement, les documentalistes ont aussi une mission de service aux équipes : ils peuvent, à la demande des agents,

effectuer des recherches parmi la documentation interne à l'Institut mais aussi dans les ressources d'autres institutions. Pour ce faire ils travaillent en collaboration avec leurs collègues de I'Inrap et les structures documentaires spécialisées en archéologie externes à I'Institut.

Gestionnaire de documentation Metz Gestionnaire de documentation Amiens Gestionnaire de documentation La Courneuve Gestionnaire de documentation Orléans Gestionnaire de documentation Tours

Gestionnaire de documentation Cesson-Sévigné Gestionnaire de documentation Carquefou Gestionnaire de documentation Pessac Gestionnaire de documentation Saint-Orens Gestionnaire de documentation Nîmes Gestionnaire de documentation Bron

Gestionnaire de documentation Clermont-Ferrand

Gestionnaire de documentation Dijon

Centre archéologique doté d'un centre de documentation

- Autre centre archéologique doté d'un fonds documentaire

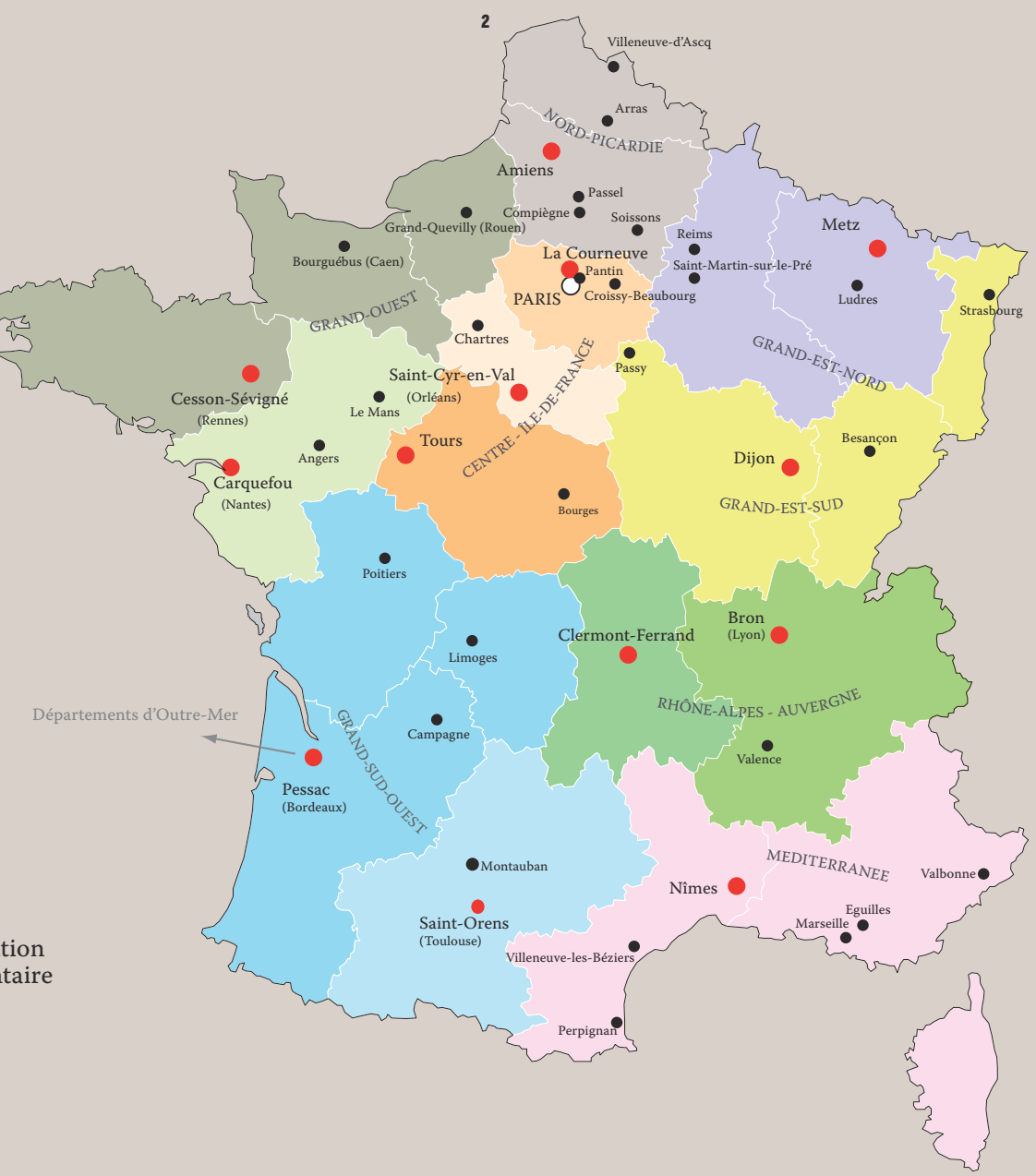

3.a Diagnostics

$3 \%$

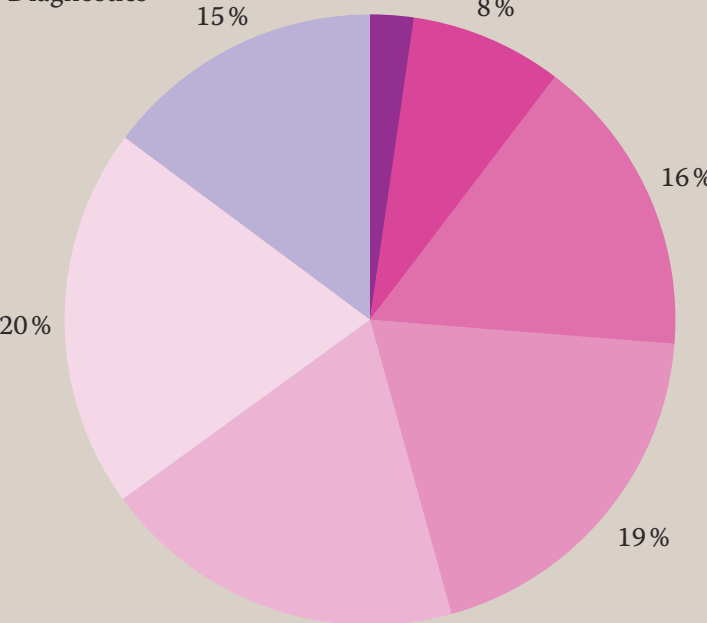

$19 \%$

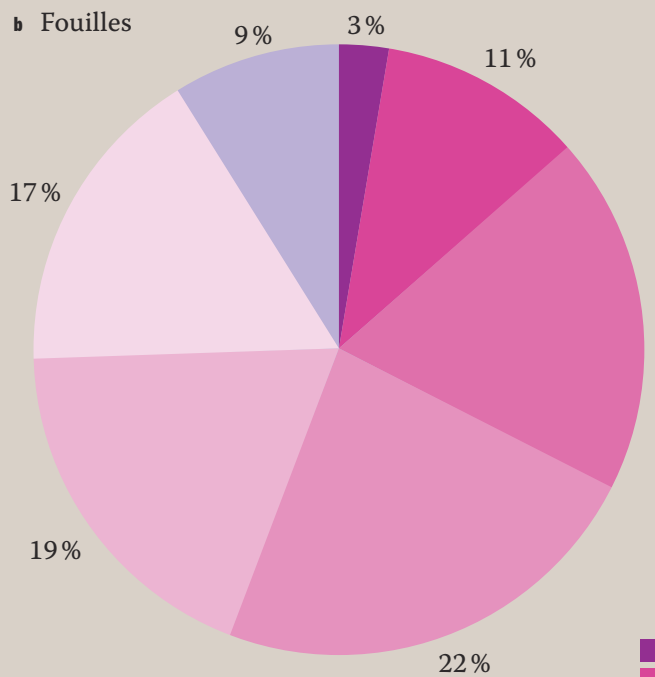

$19 \%$

Paléolithique

Néolithique

Protohistoire

Antiquité

Époque médiévale Époque moderne

Époque contemporaine
a. Répartition des rapports
du catalogage du rapport.
Si plusieurs descripteurs
de diagnostics catalogués
dans Dolia par thèmes
chronologiques.
b. Répartitions des rapports
de fouilles catalogués dans
Dolia par thèmes chronologiques.
Les graphiques sont établis
à partir de l'indexation
chronologiques peuvent être
attribués à un même rapport,
un gestionnaire de
documentation peut choisir
de ne pas signaler une période
dont les vestiges sont signalés
comme anecdotiques dans
effectuée par les gestionnaires
le rapport.

de documentation lors 
opérations, en raison encore de sa valeur scientifique. Au-delà du terrain, ces rapports sont la source primaire des travaux de recherche qui ont à mobiliser les données au plus près de leur source et doivent pouvoir en identifier les producteurs. Document quasi emblématique de la profession, le rapport d'opération sous une seule appellation cumule en fait plusieurs exercices et, par là, plusieurs fonctions : rapport d'activité, document contractuel, expertise, publication « primaire » du site... [cf. encadré p. 76].

Ce corpus exceptionnel de documents méritait donc d'être organisé, classé, signalé et mis à disposition. Ainsi valorisé, chaque rapport, loin de n'être qu'un simple « document administratif », prend pleinement sa place dans une « documentation scientifique » référencée et sur laquelle s'appuiera une recherche. Plus particulièrement, les rapports de fouilles, régulièrement évalués par les commissions régionales de la recherche archéologique (Cira), devraient être appelés rapidement à obtenir un autre statut : l'Agence d'évaluation de la recherche et de l'enseignement supérieur avait considéré que les rapports de fouilles appartenaient à la production scientifique ${ }^{3}$ des unités de recherche ; il est désormais monnaie courante de les voir cités aux côtés « d'autres » publications dans les bibliographies. Parions qu'ils devront prochainement être pris pleinement en compte dans l'évaluation de la carrière des chercheurs eux-mêmes.

Essentiellement, Dolia n'a pour l'instant concerné que le fonds des rapports d'opération produits par l'Inrap. Beaucoup reste à faire, à commencer par achever l'enregistrement du fonds, reconstituer un fonds $\mathrm{Afan}^{4}$ et prendre en compte les rapports d'opération des autres opérateurs de l'archéologie préventive, tel que le prévoit la législation (art. L 523.11 du Code du Patrimoine). Méritera aussi d'être enregistrée et signalée dans Dolia la littérature dite « grise » mais essentielle à la recherche : rapports en tous genres, techniques et scientifiques, rapports d'activité, le tout constituant un fonds professionnel d'archéologie préventive.

Car ces documents recèlent un savoir opérationnel et scientifique qui réclamerait le plus souvent, sauf naturellement confidentialité expresse, une diffusion autre qu'une obligation de dépôt en fin de mission.

3. Agence d'évaluation de la recherche et de l'enseignement supérieur, Critères d'évaluation des entités de recherche le référentiel de l'AÉRES, p. 11,36 .

4. L'Association pour

les fouilles archéologiques nationales, à partir des années 1980 , a réalisé la plupart des opérations d'archéologie préventives. À sa création, en 2002, l'Inrap a hérité des rapports d'opération produits par l'association.

\section{"Archives de fouille », documentation scientifique}

Parmi la documentation qui reste encore sousles « archives de fouille», c'est-à-dire l'ensemble des documents à caractère scientifique - excluant par là les documents administratifs - produits à l'occasion d'une opération, et ceci quels que soient le degré d'élaboration du message porté par le document (minute, brouillon, état intermédiaire ou achevé), le vecteur d'expression du message (écrit ou iconographique) et le support ou la technique mise en œuvre (numérique, argentique, graphique...). Si réglementairement la documentation originale doit être versée à l'État, le renseignement qu'elle porte reste un complément indispensable aux ensembles de mobilier et aux rapports d'opération. Globalement, ces lots issus de l'activité de terrain méritent donc d'être mieux mis à la disposition des chercheurs plutôt que de rester voués à un archivage « fermé ».

Pour l'Inrap, il s'agit là d'un nouvel enjeu documentaire : organiser des fonds scientifiques, nonobstant la remise de ces archives à l'État qui n'en n'empêche pas une duplication exhaustive ou partielle. Et on peut s'attendre à ce que l'inexorable « tout numérique » contribue à en faciliter la démarche, même si, pour l'heure, il conduit souvent au foisonnement, à la démultiplication des documents-fichiers et à la disparité des formats natifs. Mais l'enjeu est crucial et demandera expertises, expérimentations, concertation interne, collaborations externes avec des réseaux professionnels : l'organisation des tâches et la définition de protocoles partagés dans le domaine du dessin et de l'infographie, le déploiement de systèmes d'informations géographiques, sont des opportunités d'unifier les formats au stade de la production, tandis que le catalogue Dolia est quant à lui apte à enregistrer ces fonds. Et quoi qu'il en soit, une partie du chemin est déjà parcourue avec l'obligation de signaler chaque document au sein des inventaires prévus par l'arrêté du 27 septembre 2004 et intégrés au rapport d'opération pour les deux inventaires propres à identifier les éléments archéologiques, contextes et mobilier [cf. encadré p. 78]. Si les catégories de ces différents inventaires apparaissent aujourd'hui dépassées, le principe d'un catalogue documentaire attaché systématiquement à une opération est là ; reste à lui donner une autre ambition que celle de figurer en tableau annexé à un produit papier.

\section{Le système documentaire de l'Inrap}

Il ne pouvait être question de gérer les fonds documentaires de l'Inrap sans que soit mis en place un outil unique permettant a minima le signalement, le référencement et la localisation des documents conservés par l'établissement, qu'il s'agisse d'ouvrages édités acquis ou de littérature grise produite par les équipes. Plusieurs arguments ont prévalu dans le choix de la solution informatique. En tout premier lieu, le désir de gérer l'ensemble de la documentation scientifique de l'établissement avec un outil unique adapté aux compétences du réseau de documentalistes mis en place, et le souhait d'inscrire le projet dans un cadre existant par ailleurs, de manière à pouvoir développer le catalogue rapidement, et l'ouvrir à d'autres. En second lieu, la nécessaire interopérabilité, notamment avec les autres catalogues documentaires relatifs à l'archéologie, mais également la contrainte d'une alimentation et d'une consultation à distance impliquant le choix d'une solution client-serveur. Enfin, la possibilité de pouvoir rendre accessible depuis la « notice 
Le rapport d'opération, un document de choix pour l'Inrap

Document à juste titre exigé par l'छ́tat à l'issue de chaque excavation archéologique contrôlée, le « rapport » reste pour l'opération et pour le site qui s'y associe le document le plus exhaustif et polyvalent dont l'administration et la recherche archéologiques disposeront ; car en dépit de l'unicité de sa désignation, plusieurs genres d'exercices sont en réalité menés dans le rapport. Pour les rapports de fouilles au sens strict, il s'agit bien d'un document venant clore une opération - un acte administratif qui s'achève avec la validation de l'autorité prescriptrice -, mais le rapport est aussi un document patrimonial qui conserve par l'image et par l'écrit ce qui a disparu ou a été déplacé, principe particulièrement fort puisqu'il justifie en grande partie toute l'archéologie, préventive ou non. Le rapport est également un compte rendu d'activité d'une équipe sur un chantier. Mais aussi, peut-être surtout, le rapport de fouilles constitue toujours un document initial d'analyse archéologique qui rend compte d'observations et de réflexions inédites, en somme une publication primaire du site auquel il se rapporte, caractère d'autant plus important que bien des sites, même majeurs, ne demeureront connus que par le rapport de fouilles dont ils ont été l'objet. Quant au rapport de diagnostic, s'il répond aux mêmes visées, sa vocation est de surcroît d'être une expertise menée à des fins d'évaluation d'un potentiel archéologique qui conduira - ou non - à procéder à des fouilles. Il est donc d'autant plus important quand le diagnostic n'est pas suivi de fouilles...

C'est dire tout l'intérêt scientifique de ces documents, en outre encadrés - une spécificité française - pour l'archéologie préventive par une réglementation positivement exigeante (arrêté du 27 septembre 2004 portant définition des normes de contenu et de présentation des rapports d'opérations archéologiques ; signalons que l'archéologie programmée est en passe de s'aligner sur cette réglementation) et qui font l'objet de validations administrative et scientifique de la part des services régionaux de l'archéologie (Drac/SRA) et des commissions interrégionales de la recherche archéologique (Cira).

Prenant en compte les nombreuses réflexions dont il a fait l'objet depuis plus de vingt ans (dont on trouvera les références dans $\mathrm{Ph}$. Soulier [dir.], Le Rapport de fouille archéologique, Paris, De Boccard, 2010), considérant son intérêt scientifique et professionnel, 1'Inrap a donc fait du rapport l'objet prioritaire de son dispositif documentaire, en favorise la diffusion et en optimise la production et la conception. Ainsi, à partir de l'année 2009, 1'Inrap a unifié la présentation de ses rapports : 1'adoption d'une maquette unique a contribué à afficher publiquement le caractère national de l'établissement permettant ainsi d'identifier clairement ses productions parmi celles des autres opérateurs.

Cette unification allait de pair avec une réflexion de fond s'appuyant sur l'arrêté du 27 septembre 2004 et relative au contenu, à la conception et la structure des rapports. Toujours en cours, cette réflexion repose donc sur les multiples natures du rapport (administrative, patrimoniale, opérationnelle et scientifique); il s'agit d'ordonner les façons de rendre compte des travaux, des observations et des interprétations archéologiques. Nonobstant les obligations propres à la section I de l'arrêté du 27 septembre 2004, quelles sont les pièces indispensables permettant d'atteindre ces objectifs ? Quel jeu de plans peut rendre compte des emprises, des excavations, des vestiges, des emplacements de coupes, de prélèvements, etc. ? Dans quelle mesure aussi les données rassemblées pour l'opération (carte archéologique, données géologiques) doivent figurer dans le rapport ? Quelle part accorder aux données propres aux formations superficielles ? Quels formats numériques de constitution et/ou de diffusion? Concernant le corps même du rapport, la question de « logique de l'exposé » est naturellement centrale. Le choix peut se réduire à deux démarches : suivant l'ordre opérationnel, par zone, par excavation (tranchées, sondage), ou par occupation, par période chronologique, par phase. Description, figuration, énumération des vestiges, traces, structures présentes dans le rapport ne sauraient aller de soi : degré de détail, exhaustivité ou échantillonnage concernant des vestiges singuliers ou répétitifs, demeurent toujours à raisonner. Si le signalement complet des structures peut être renvoyé réglementairement à la section III du rapport, consacrée aux « inventaires techniques », ces derniers pourraient constituer - pour les structures archéologiques au moins - de véritables catalogues détaillés. Infin, plus spécifiquement pour le rapport de diagnostic, comment rapporter - ou non des observations menées sur des terrains archéologiquement pauvres ou stériles? Naturellement, ces questions de fond nécessitent complémentairement une réflexion plus éditoriale destinée à optimiser l'usage et normaliser la forme des « vecteurs d'information » propres à n'importe quel document imprimé et qui doivent s'associer pertinemment pour construire un discours : textes, images, symboles, tableaux, infogrammes, figures. À l'ensemble de ces questions devrait répondre, à moyen terme, la rédaction d'un « précis » consacré à la conception, rédaction, édition des rapports d'opération en archéologie préventive. 
descriptive » le document au format électronique, l'inventaire des fonds réalisés dans les premiers mois du projet ayant révélé l'existence de très nombreux documents numériques qu'il fallait pouvoir exploiter.

Le choix d'un système intégré de gestion de bibliothèque (SIGB) propriétaire, ${ }^{\mathbf{5}}$, respectant tout d'abord le standard international Unimarc, format officiel d'échange de l'information bibliographique, et apte à décrire tout type de document - monographie et article, carte, photographie, base de données ou document audiovisuel - s'est imposé. Il devait par ailleurs rendre possible la consultation en texte intégral des documents signalés.

\section{Signalement, description}

L'Unimarc permet à la fois de décrire la forme - titre, auteur, édition, collation, etc. - et le contenu d'un document via un résumé, une indexation, mais aussi, il comporte un certain nombre de champs libres que le catalogueur peut utiliser pour des renseignements propres à son contexte de travail. Via ces champs 9oo, il a été décidé de décrire sommairement le contexte des opérations archéologiques - localisation, responsable d'opération, numéro d'enregistrement de l'opération dans le système de gestion de l'activité de l'Inrap - dont rendent directement compte une partie des documents référencés dans Dolia. Sans être une base de données des opérations de terrain, et conservant le document comme objet principal, Dolia contient ainsi le minimum d'informations nécessaires sur les opérations archéologiques pour pouvoir être mis en relation avec les autres systèmes d'information de l'établissement.

Précisons que, créé pour la description bibliographique des documents édités, le format Unimarc ne s'oppose en rien à la description de la littérature grise et des « archives de fouille» à condition que celles-ci répondent à un certain nombre de « critères éditoriaux » propres au référencement. À ce titre, le rapport d'opération - comportant une couverture et/ou une page de titre sur lesquelles sont mentionnés le ou les auteurs, le titre, souvent composé à partir de la localisation géographique de l'opération, et une année d'édition - ne pose aucun problème majeur pour prendre place dans un catalogue respectant des normes internationales. L'exercice est d'autant plus aisé que le rapport d'opération est un document normalisé qui a fait l'objet de nombreuses recommandations éditoriales, les principales étant la circulaire dite « DFS » de $1993^{\mathbf{6}}$, et plus récemment l'arrêté de septembre 2004 relatif aux rapports d'opération ${ }^{7}$. En 2009, l'Inrap a unifié la présentation de ces documents en diffusant une maquette répondant à des normes de présentation proches de celles d'une publication scientifique. Conçue dans le respect des textes réglementaires, elle a également tenu compte des contraintes posées par le catalogage de cette littérature grise, et des recommandations ont notamment été émises pour la rédaction d'un « titre de contenu » concernant les rapports d'opération de fouilles, élément permettant d'identifier rapidement le sujet principal du document.

En outre, l'arrêté de septembre 2004 prévoit que les rapports d'opération comprennent une « notice scientifique résumant les principaux résultats de l'opération ${ }^{\mathbf{8}}$ ainsi que des mots-clés issus des thésaurus « Chronologie » et «Interprétation » de la base de données nationale Patriarche, autant d'éléments participant au catalogage. En effet, le contenu des documents est décrit dans le système documentaire de l'Inrap, d'une part au travers d'un résumé de l'œuvre - dans le cas des rapports d'opération, il peut être établi par les documentalistes sur la base des notices scientifiques réalisées par les archéologues et d'autre part, chaque notice de Dolia exige une indexation en langage contrôlé à partir des microthésaurus «Lieux », « Sujets », «Chronologies » et « Peuples » du thésaurus Pactols [cf. encadré p 78] dans lesquels les mots-clés indiqués au sein des rapports trouvent bien évidemment leurs équivalents. Lors du choix d'un thésaurus en 2006 , deux possibilités s'offraient à l'Inrap : le thésaurus Pactols, les thésaurus de la base de données nationale Patriarche. Si les seconds sont déjà employés dans la première section des rapports pour caractériser l'opération et permettent une description des sites plus précise, le premier est plus riche, couvre mieux les différents aspects d'un rapport - résultats de l'opération, description du mobilier, études spécialisées, études documentaires et d'archives - et est plus adapté à la description des ressources éditées. Le caractère évolutif du thésaurus Pactols a levé les dernières hésitations, puisqu'il devenait envisageable d'enrichir le thésaurus des termes qui pourraient manquer? L'utilisation d'une liste fermée de termes liés entre eux par des relations sémantiques et hiérarchiques pour la description des documents et l'interrogation du catalogue peut apparaître comme une contrainte. En effet, elle oblige à se plier aux choix des concepteurs du thésaurus et ce parfois au détriment des usages en vigueur localement. Cependant, utilisé dans le cadre d'un catalogue d'envergure nationale alimenté par plusieurs documentalistes, pour décrire des réalités parfois proches mais nommées différemment suivant les régions, le thésaurus renforce la cohérence de la base de données en contribuant à l'unification des descriptions et facilite la mise en relation des documents [ill. 3]. À condition d'en connaître un minimum la structure, il peut être une aide à l'énoncé des recherches.

\section{Spécificité des documents d'archives}

Si le référencement des rapports d'opération ne pose aucun problème majeur, comme le prouvent les quelque 15 ooo notices de rapport de diagnostic et de fouilles accessibles dans Dolia, le catalogage par Ever Team
6. Circulaire du

9. Depuis 2006,

partenaires a contribué

à développer au sein

les vocabulaires de

l'archéologie urbain

et de l'archéologie

médiévale, modern

et contemporaine. 
Des ressources d'avenir à valoriser : les inventaires réglementaires

L'arrêté du ¿'7 septembre 2004 prévoit que chaque rapport d'opération comprenne une section III comprenant 7 inventaires techniques. Ces inventaires doivent récapituler, identifier et plus ou moins caractériser deux catégories d'éléments mis en série, les uns documentaires (clichés, dessins, carnets...) et qui ressortissent plus à la gestion des archives de la fouille, les autres archéologiques et qui concernent directement les « données " archéologiques ; deux inventaires particulièrement riches d'informations, celui des unités stratigraphiques et structures archéologiques, et celui du mobilier. Pour le premier, l'arrêté précise seulement que les relations stratigraphiques devront être mentionnées, pour le second en revanche il est bien plus disert : ordonnancement par catégorie, unité d'enregistrement, possibilité de procéder par lot, lieu et état de conservation... L'intérêt de ces inventaires reste à être mieux compris, voire à être explicité. t.t l'obligation qu'ils figurent - imprimés - dans le rapport d'opération est peut-être un peu " courte » comme rentabilité scientifique et possibilité d'exploitation. Car il s'agit là des seuls documents où est mentionnée l'exhaustivité des « données » archéologiques caractérisées et observées sur un site : en exiger une forme informatisée aussi simple soit-elle mais indépendante du rapport serait un bien. Mieux encore serait d'en organiser une sorte de dépôt légal, antichambre à une plateforme de données archéologiques issues de l'archéologie préventive. Ce qui suppose naturellement qu'au moins soit établie ou achevée en préalable une organisation par rubriques de ces inventaires et qu'en soit affirmé le statut de donnée publique.

Enfin, au sein du rapport d'opération, ces deux inventaires pourraient constituer, mieux que de simples annexes, de véritables catalogues raisonnés, détaillés et illustrés s'il le faut, comprenant les séries exhaustives des structures ou mobiliers échantillonnés dans la section II tout en allégeant celle-ci de fastidieuses descriptions.

\section{Le réseau Frantiq}

Frantiq est un réseau documentaire spécialisé en archéologie, de la Préhistoire à l'époque contemporaine (wwww.frantiq.fr). Organisé en Groupement de service du CNRS depuis 2010 (GDS 3378) adossé à la Maison archéologie et ethnologie René-Ginouvès de Nanterre (IMAF), il est piloté entre autres par le « Service d'ingénierie documentaire et réseaux pour l'archéologie " (SIDRA) de la MAF et la plateforme technologique « Têtes de réseaux documentaires » (TRD) de la Maison de l'Orient et de la Méditerranée (MOM) à Lyon. La participation à la fédération se fait sur la base du volontariat soit par simple lettre d'adhésion pour les unités de recherche du CNRS soit par convention pour les autres partenaires (collectivités territoriales, musées nationaux, Inrap). . En 2012 , une convention a même été signée avec la sousdirection à l'archéologie du ministère de la Culture et de la Communication pour l'intégration des services régionaux de l'archéologie, du Drassm et du Centre national de Préhistoire. Frantiq compte aujourd'hui une quarantaine de membres, acteurs de l'archéologie préventive comme programmée, et répartis sur l'ensemble du territoire national. Depuis plus de trente ans, Frantiq développe, dans le respect des normes et standards internationaux de la bibliothéconomie et des ontologies, un catalogue bibliographique commun aux différents partenaires et un thésaurus spécialisé destinés à la communauté scientifique et académique. Le catalogue collectif indexé (CCI) contient plus de 400000 notices de documents divers : ouvrages et articles scientifiques parmi lesquels figurent des documents rares du fait de la spécificité de certains fonds, catalogues d'exposition, rapports d'opérations archéologiques. Il assure le signalement et la localisation des ressources physiques et, de plus en plus, permet 1'accès à la ressource en ligne lorsque celle-ci existe ; il pointe actuellement sur le portail Persée et tend à s'associer avec le réservoir Revues.org. Les notices du CCI sont indexées avec le thésaurus Pactols construit et régulièrement mis à jour par la fédération Frantiq. Traitant de l'archéologie de la Préhistoire à la Grande Guerre, ainsi que de l'Antiquité sous tous ses aspects (à l'origine Frantiq réunissait essentiellement des unités de recherche spécialisée dans les sciences de l'Antiquité), Pactols est organisé en sept microthésaurus -« Peuples ", « Anthroponymes ", "Chronologie ", « Toponymes ", " CFuvres ", « Lieux », « Sujets » - qui respectent les normes des thésaurus polyhiérarchiques et multilingues. Ce thésaurus est géré par le logiciel libre OpenTheso Web développé par la TRD. Dans un premier temps, la participation de l'Inrap à la fédération Frantiq s'est faite par le biais de la convention cadre qui unit l'Inrap et le CNRS. Depuis la création du GDS, une convention de partenariat précise les obligations de chacun : l'Inrap s'engage à enrichir le catalogue collectif indexé des données bibliographiques, normalisées et indexées, propres aux rapports d'opérations archéologiques qu'il conduit, participe à l'enrichissement du thésaurus Pactols et prend part aux groupes de travail du GDS. Fn contrepartie, le GDS met à la disposition de l'Inrap le catalogue des bibliothèques, l'outil d'indexation et le site web du réseau ainsi que le thésaurus Pactols. 
d'une sélection d' « archives de fouille », prévu initialement, s'avère une tâche bien plus délicate. Bien que composée de documents communément désignés sous l'appellation d'archives de fouilles ${ }^{\mathbf{1 0}}$ puisqu'ils conservent la mémoire d'une opération, cette sélection ne peut pas faire l'objet d'un traitement archivistique au sens strict du terme. Celui-ci repose sur la notion de respect organique $\mathrm{du}$ fonds (respect de la provenance, respect de l'intégrité du fonds et de son ordre originel, respect des normes et usages de la conservation préventive) qui ne saurait être mise en œuvre dans Dolia puisque ne sera conservée qu'une sélection de documents, choisis pour l'intérêt de l'information scientifique qu'ils véhiculent. Ces documents entreront dans la chaîne documentaire mise en place pour le traitement des rapports : collecte, traitement intellectuel du document en vue de l'établissement de notices bibliographiques dans Dolia, classement et conditionnement pour mise à la disposition des agents de l'Inrap.

Souvent conçues comme des documents de travail à destination au mieux de l'équipe, au pire du seul responsable d'opération, la plupart de ces archives ne présentent pas les éléments indispensables à leur citation et référencement : un titre, un auteur, une année, etc. De ce fait, le catalogage à l'unité apparaît comme une gageure peu raisonnable et ceci quelle que soit la sélection effectuée sur l'ensemble de la documentation scientifique versée à l'État à l'achèvement d'une opération. Pour pallier cette difficulté, Dolia a été paramétré de façon à pouvoir signaler et décrire tout autant un document (image, texte, etc.) individualisé qu'un lot de documents de même nature selon le principe des liasses utilisées dans la gestion des archives. L'intégration de ce nouveau fonds, collecté de façon souvent hasardeuse auprès des archéologues, est une expérience qu'il faudra mener concrètement dans les prochains mois ; elle s'accompagnera forcément de recommandations relatives au signalement des documents.

\section{Localisation, accessibilité}

Au-delà d'être une base de données permettant de signaler et de présenter la documentation scientifique de l'Inrap, Dolia, comme de nombreux catalogues documentaires, renseigne sur le nombre d'exemplaires, leur nature, leur localisation, leur disponibilité, etc. Le réseau documentaire de l'Inrap s'est dès lors doté d'outils d'aide à la description des exemplaires physiques : plan de classement et code-barres. Si le code-barres n'est qu'un numéro d'ordre servant à identifier les exemplaires, permettant le récolement du fonds et le prêt de documents aux agents et entre centres de documentation, le plan de classement quant à lui génère une cote propre à chaque document qui, tout en facilitant l'organisation physique du fonds, en précise le contenu. Deux plans de classement, élaborés selon les principes d'une organisation des connaissances non pas hiérarchique mais thématique, ont été adoptés pour coter et ordonner les fonds. En toute logique et dans le respect des habitudes de consultation, la notion d'opération - lieu, date, auteur du rapport - prévaut dans le classement des rapports d'opération tandis que les œuvres éditées sont rangées selon des critères géographique, chronologique et thématique.

Cependant, la part croissante des documents électroniques et les enjeux du projet documentaire défini en 2006, à savoir le signalement public de la documentation scientifique présente à l'Inrap et sa mise à disposition en interne, ont été déterminants pour le choix du système : il devait impérativement permettre la gestion électronique de documents. D'autant plus que la généralisation de l'informatique dans l'acquisition, le traitement et la mise en forme des données archéologiques vient aujourd'hui bouleverser les notions de données brutes, primaires, descriptives, interprétatives, synthétiques, mais également d'original, de copie, de reproduction, d'impression, de numérisation, etc. Si l'exploitation d'une sélection d'archives de fouilles au format numérique est un chantier ambitieux qui n'en est encore qu'à ses prémices, la mise en ligne des rapports d'opération en PDF, selon des critères différents, qu'il s'agisse d'une mise à disposition publique ou interne, était une priorité dès 2006. Cette action a nécessité un important travail préalable de reconstitution du fonds, l'objectif étant à terme de rendre accessible en ligne, à tous les agents de l'Inrap, l'ensemble des rapports de diagnostic et de fouilles produits par l'établissement. Elle s'accompagne d'une nécessaire réflexion sur la gestion des fichiers numériques, aussi bien sur la manière de les générer, format d'enregistrement, définition, sécurisation, que de les identifier, nommage et métadonnées, ou encore de les conserver. Ce travail, en cours mais à des degrés d'avancement différents selon le type de document, invite l'Inrap à collaborer avec des organismes spécialisés dans ce domaine.

Lors de la mise en ligne de Dolia en 2009, dans un milieu encore très réservé face à l'ouverture des données (en anglais open data), il a été décidé que le catalogue ne serait accessible qu'après « identification ». Les agents de l'Inrap accédaient au catalogue grâce à leurs paramètres d'identification utilisés pour le site Intranet de l'Inrap, tout autre utilisateur était invité à s'inscrire via un formulaire en ligne ; il recevait automatiquement un mot de passe lui permettant de consulter les notices du catalogue et une sélection de rapports de fouilles au format PDF. La mise en ligne répondait à des critères déontologiques et de qualité ; elle devait, de plus, avoir été autorisée par l'auteur, après confirmation par ce dernier qu'aucun projet de publication n'était programmé dans un délai de cinq ans.

Les réflexions menées actuellement sur l'accès à l'information, ainsi que le développement des nouvelles technologies, ont invité l'établissement 
à modifier depuis peu sa pratique : les notices du catalogue sont en accès libre pour tous ; parallèlement, l'accès authentifié unique jusqu'en juin 2012 a été dédoublé afin de permettre un accès différencié pour les agents de l'Institut. Ceux-ci peuvent consulter via Dolia l'ensemble des rapports d'opération, de diagnostic et de fouilles, qui sont progressivement attachés aux notices depuis l'été 2013 ; les autres utilisateurs continuent à accéder à une sélection de rapports de fouilles établie selon les critères évoqués précédemment et précisé sur le site de l'Inrap (http://www.inrap.fr).

\section{Moteur de recherche, résultats}

Le catalogue s'ouvre sur un moteur de recherche simple qui permet, via un champ de recherche unique, d'interroger un index alimenté par les valeurs saisies dans les zones titre, auteur, résumé, indexation libre et sujet. La requête est formulée comme une juxtaposition de termes combinés par défaut par l'opérateur booléen restrictif « et » ${ }^{\mathbf{1 1}}$ qui peut cependant être remplacé, à condition qu'il soit inscrit, par l'un des deux opérateurs suivants : « ou » ou « sauf ». Ce moteur de recherche simple est utile pour retrouver un document connu, mais aussi pour appréhender un sujet, les recherches étant rarement totalement infructueuses.

Pour faire une recherche plus précise, le catalogue dispose d'un moteur de recherche dite « experte» qui permet d'interroger, séparément ou en les combinant avec les opérateurs booléens, la plupart des zones de description d'un document, qu'il s'agisse du signalement (titre, auteur, éditeur, collection) ou du contenu (résumé, indexation). L'interrogation via ce moteur de recherche experte se fait de préférence en langage contrôlé, via les index ou les thésaurus. Plus contraignante du fait de la nécessité de trouver les termes employés dans le catalogue au risque d'ignorer des réponses, la recherche experte est aussi plus pointue.

Un moteur de recherche en texte intégral sur les documents attachés est accessible sur chacune des interfaces de recherche, simple ou experte. Il permet une interrogation en langage naturel, son utilisation est rarement infructueuse mais oblige à une lecture attentive des résultats pour en évaluer la pertinence. L'utilisateur dispose d'outils pour gérer les résultats : enregistrement des requêtes, conservation des notices dans un panier, sur des étagères virtuelles publiques ou privées, etc. De plus, Dolia est aujourd'hui compatible avec Zotero, ce qui permet, entre autres, d'exploiter les notices de rapports au même titre que n'importe quelle référence bibliographique.

\section{Perspective}

Si Dolia remplit actuellement les objectifs fixés en 2006, localiser et décrire les ressources documentaires scientifiques disponibles à l'Inrap, et permet les échanges de données avec d'autres systèmes équivalents en archéologie - le catalogue collectif indexé du réseau Frantiq par exemple -, il repose cependant sur une technologie et des normes déjà anciennes. Des évolutions sont en cours qui devraient permettre la mise en place d'un portail documentaire donnant accès à la fois au catalogue Dolia et à des produits dérivés comme les dossiers bibliographiques sur lesquels travaillent actuellement les documentalistes de l'Inrap. Surtout, de nouveaux modes d'indexation liés au développement du web 2.o sont apparus et pourraient venir en complément des thésaurus Pactols si ce n'est s'y substituer. Des systèmes comme les folksonomies, reposant sur la participation des utilisateurs qui sont invités à décrire la ressource qu'ils consultent avec leurs propres termes ou mots-clés, pourraient rendre la recherche plus intuitive.

Pour l'Inrap, Dolia s'inscrivait naturellement dans la vocation scientifique de l'établissement. Il s'agissait non seulement d'ouvrir ses portes à un public spécialisé comme à un public d'érudits non spécialistes, mais encore d'offrir aux chercheurs de l'établissement les moyens documentaires d'exercer leur métier, et enfin, de gérer en quelque sorte l'avenir en organisant la mémoire scientifique de l'Institut. Si le projet documentaire de l'Inrap ne devait être commencé qu'aujourd'hui, le document papier ne serait peut-être pas le point d'entrée du système et la solution technique ne serait donc peut-être pas du même type. Car le document numérique occupe désormais une place beaucoup plus importante qu'en 2006. Ainsi, le recours à une maquette unique de mise en page des rapports, leur envoi pour impression à un prestataire qui restitue systématiquement, avec les exemplaires papier, leurs fichiers respectifs en PDF, les campagnes de numérisation de rapports plus anciens sont autant d'éléments qui entraînent la multiplication des fichiers numériques disponibles.

Par ailleurs les mouvements conjoints d'open data et d'open access ${ }^{\mathbf{1 2}}$ apparus il y a une dizaine d'années dans les pays anglo-saxons ont donné naissance en France à une série d'initiatives visant à la mise en ligne des données publiques. L'État s'est engagé dans une politique volontariste de diffusion en ligne des documents administratifs via la mission Etalab ${ }^{\mathbf{1 3}}$. C'est dans ce cadre que l'Inrap a été sollicité par le ministère de la Culture pour mettre en libre accès une sélection de données qui pourront être réutilisées et faire l'objet de traitements automatisés. Une partie des informations relatives aux rapports d'opération contenues dans Dolia, le titre, les indexations « Sujet », « Lieu » et « Chronologie » ont ainsi été mises à la disposition du ministère.

Parallèlement, le ministère de l'Enseignement supérieur et de la Recherche ainsi que les grands établissements français de recherche mènent une réflexion autour de la mise à disposition des données de la recherche. Cela a donné naissance, d'une part au projet de Bibliothèque scientifique numérique ${ }^{\mathbf{1 4}}$, d'autre part à des plateformes 
15. Notons que l'Inrap possède depuis le second semestre de l'année 2009 un sous-portail dans le portail HAL ; toutefois, il est plus utilisé actuellement pour référencer les travaux des personnels de

l'établissement que pour mettre à disposition des documents au format numérique. http://hal-inrap. archives-ouvertes.fr/ (consultation du 28/10/2013). 16. Projet de plateforme numérique de données archéologiques s'inscrivant dans le septième programme-cadre de la Commission européenne pour la recherche et le développement technologique. Le Pin (Université de Florence) et ADS (Université de York) coordonneront pendant quatre ans (2013-2017) le travail de vingt-quatre partenaires, techniques et scientifiques. d'archives ouvertes interinstitutionnelles comme l'est $\mathrm{HAL}^{\mathbf{1 5}}$, thématiques comme la plateforme des physiciens Arxiv, ou encore institutionnelles comme la plateforme SAM de l'École des Arts et Métiers. Ces différents projets ont tous pour point commun de donner accès en ligne, avec plus ou moins de contrôle, aux productions d'une communauté de chercheurs.

C'est dans ce contexte quelque peu en ébullition, alors que se multiplient journées d'étude, rencontres, colloques et autres groupes de travail transversaux, qu'une réflexion est menée actuellement à l'Inrap pour la création d'une plateforme nationale de données archéologiques qui mettrait à disposition une documentation aujourd'hui disparate et dispersée. L'ensemble de la documentation produite au cours d'une opération d'archéologie, que ce soit des minutes de terrain, des bases de données, des inventaires réglementaires ou des rapports d'opération, serait accessible depuis une interface unique et décrit avec un système d'indexation commun. L'ambition est de faciliter la diffusion du savoir et les échanges scientifiques entre les acteurs de l'archéologie, étudiants, enseignants, chercheurs et archéologues de terrain ; une vocation, nous le répétons, inscrite dans les missions publiques de l'Inrap. Afin de voir aboutir un tel projet, l'Inrap devra préalablement mener à terme un certain nombre de réflexions : sur les conditions réglementaires et déontologiques de mises en ligne des données de la recherche en archéologie, sur l'adoption de standards propres au formatage de données numériques dans le cadre d'une plateforme nationale. Notons enfin que le projet européen Ariadne ${ }^{\mathbf{1 6}}$, auquel participe l'Inrap, fera avancer le dossier.

Alors, si le projet d'origine semble abouti, il ne saurait se figer dans la satisfaction du devoir accompli et un confort routinier d'alignement infini de références. Aussi, doit-on s'attendre à ce que le projet Dolia, dépassant le cadre du catalogue documentaire et de la bibliothèque numérique, vienne s'inscrire dans un « système d'information documentaire d'archéologie préventive " pour un partage systématique de données - somme toute - publiques. 